\title{
Pseudotumor inflamatorio del bazo. Revisión y aportación de un nuevo caso
}

\author{
F. L. LADO LADO, M. PÁRAMO DE VEGA, J. A. TORRE CARBALLADA, A. LOIS \\ PERNAS, I. ABDULKADER ${ }^{1}$
}

Servicios de Medicina Interna $y^{1}$ Anatomía Patológica. Departamento de Medicina. Hospital Clínico Universitario de Santiago. Santiago de Compostela, A Coruña

INFLAMMATORY PSEUDOTUMOR OF THE SPLEEN. REVIEW AND A NEW CASE REPORT

\section{RESUMEN}

El pseudotumor inflamatorio de bazo definido como una lesión tumoral reactiva, que muestra un espectro de cambios reparativos o inflamatorios inespecíficos de comportamiento benigno es una entidad rara que por sus características puede dar lugar a confusión con otras patologías, especialmente procesos de tipo linfoproliferativo. Si bien, es de origen desconocido, entre sus posibles causas se han involucrado agentes infecciosos como el virus de Epstein-Barr. Presentamos el caso de un paciente de 29 años con antecedentes recientes de mononucleosis infecciosa que a raíz de presentar cuadro clínico de fiebre con pérdida de peso y esplenomegalia, después de la práctica de esplenectomía fue diagnosticado de pseudotumor inflamatorio de bazo. Se comentan las peculariedades clínicas y diagnósticas de esta entidad haciendo hincapié en sus características anatomopatológicas.

PALABRAS CLAVE: Pseudotumor inflamatorio. Bazo. Virus de Epstein-Barr.

\begin{abstract}
Splenic inflammatory pseudotumour has been defined as a benign tumour reactive lesion with unspecified reparative or inflammatory alterations. It is a mimicking tumour often confused with other pathologies, primarily, lymphoproliferative disorders. Though its aetiology remains unknown, these tumours are often associated to infectious agents such as the Epstein-Barr virus. We describe a case of a 29 year-old patient with a history of infectious mononucleosis, followed by fever, weight loss, and splenomegaly. Splenectomy detected a homogeneous, whitish $2 \mathrm{~cm}$ node diagnosed as splenic inflammatory pseudotumour. The clinical features and diagnosis of this disorder are discussed in relation to the histopathological findings.
\end{abstract}

KEY WORDS: Inflammatory pseudotumor. Spleen. Epstein-Barr virus.

Lado Lado FL, Páramo de Vega M, Torre Carballada JA, Lois Pernas A, Abdulkader I. Pseudotumor inflamatorio del bazo. Revisión y aportación de un nuevo caso. An Med Interna (Madrid) 2004; 21: 279-282.

\section{INTRODUCCIÓN}

El pseudotumor inflamatorio se define como una lesión tumoral reactiva, que muestra un espectro de cambios reparativos o inflamatorios inespecíficos de comportamiento benigno. Bajo esta denominación se incluyen una variedad de procesos que engloban las lesiones reparativas postoperatorias, los tumores como el tumor de células dendríticas y neoplasias más agresivas tal es el caso del tumor miofibroblástico inflamatorio (1-4).

Si bien, el pseudotumor inflamatorio, es reconocido en órganos como el pulmón, ganglios linfáticos, tracto gastrointestinal e hígado, es raro en el bazo (5-7). Así, en una revisión realizada en MEDLINE (palabras clave: pseudotumor/pseudotumour, spleen, inflammatory) entre los años 1984 y 2002, hemos encontrado alrededor de 79 casos, adquiriendo relevancia clínica en el año 1984 a raíz de la descripción de 2 casos por Coteligam y Jaffe (8).

Recientemente, hemos tenido la oportunidad de diagnosticar un caso de pseudotumor inflamatorio del bazo en un paciente joven que, con anterioridad, había tenido un episodio de mononucleosis infecciosa.

\section{CASO APORTADO}

Se trataba de un varón de 29 años con antecedentes de dos ingresos por síndrome febril en los últimos dos años. En una ocasión, en junio de 1999 fue diagnosticado de mononucleosis infecciosa con serología positiva para VEB (VCA IgG, VCA IgM y EBNA positi-

Trabajo aceptado: 17 de diciembre de 2003

Correspondencia: F. L. Lado Lado. Servicio de Medicina Interna. Hospital Clínico Universitario. 15706 Santiago de Compostela. A Coruña. e-mail: flladol@usc.es 
vas). En la otra, en septiembre de 2000 tras presentar cuadro clínico de 10 días de evolución de fiebre con escalofríos, astenia y malestar general con persistencia de serología positiva para VEB, y se apreció, en la ecografía abdominal el hallazgo de esplenomegalia con lesión hipoecoica que, tras responder favorablemente al tratamiento antibiótico (cobertura de amplio espectro), fue dado de alta con el diagnóstico de absceso esplénico. Desde entonces se mantuvo asintomático hasta que reingresó en junio de 2001 por un cuadro febril, de una semana de evolución, acompañado de escalofrios, cefalea, odinofagia y pérdida de peso, sin otra sintomatología acompañante. En la exploración física destacaba $\mathrm{T}^{\mathrm{a}} 39^{\circ} \mathrm{C}$, afectación del estado general, palidez cutáneo mucosa y esplenomegalia. En la analítica presentaba datos de anemia normocítica (Hb 11,9 g/dl, Hto 35,8\% y VCM $91,8 \mathrm{fl}$ ), trombopenia (44.000 plaquetas $/ \mathrm{mm}^{3}$ ), leucocitos normales $\left(7.440 / \mathrm{mm}^{3}\right)$ con neutrofilia (77\%) y desviación izquierda (7\% Cay), ligera elevación de transaminasas (GPT 48 UI/L) y enzimas de colestasis (GGT 88 UI/L, FA 232 UI/L). El resto de los parámetros bioquímicos, el estudio de la coagulación y el análisis elemental de orina fueron normales. El proteínograma, la determinación de inmunoglobulinas y el estudio inmunológico fueron normales. Se realizaron diversos estudios microbiológicos que incluyeron serologías de virus de la hepatitis (A, B y C), retrovirus (VIH-1 y 2), tifoparatíficas, brucella, mycoplasma, legionella (con la determinación de antígeno en orina), herpes, CMV, y toxoplasma que fueron negativas. Los resultados de la serología para VEB fue de IgG anti VCA $\mathrm{IgG}$ e IgG antiEBNA positivas e IgM antiVCA negativa. El resto de estudios microbiológicos (hemocultivos, cultivos de esputo y urinocultivos, que abarcaron el estudio de mycobacterias) resultaron negativos. En las pruebas de imagen, concretamente en la TAC abdominal, se evidenció una lesión focal esplénica (que se relacionó con absceso o infarto), ganglios aumentados en número a nivel paraórtico y adenopatías mesentéricas. En otros estudios, los resultados (microbiológicos e histopatológicos) obtenidos de biopsia hepática y de médula ósea dieron negativos.

Ante la sospecha diagnóstica inicial de absceso intraabdominal se inició tratamiento antibiótico con ceftazidima, metronidazol y vancomicina, evolucionando con leve mejoría clínica. Posteriormente, dados los antecedentes y el cuadro clínico comentado, y con el fin de descartar, entre otras posibilidades diagnósticas, un proceso linfoproliferativo, se procedió a la realización de laparotomía diagnóstica bajo anestesia general. En dicha intervención, se encontró una importante esplenomegalia, engrosamiento focal de la cápsula hepática de lóbulo hepático izquierdo, un pequeño nódulo peritoneal en el mesenterio del ileon distal así como múltiples y pequeñas adenopatías al mismo nivel obteniéndose muestras de todas ellas y efectuándose esplenectomía.

El estudio histológico de los ganglios indicó una linfadenitis reactiva en los ganglios linfáticos, de granuloma calcificado en nódulo peritoneal y sin hallazgos relevantes en la muestra de lóbulo hepático izquierdo. La pieza de esplenectomía, que pesaba 442 gramos y medía 15 x $11 \mathrm{~cm}$ de diámetros mayores, presentaba un nódulo subcapsular de $2 \mathrm{~cm}$. de diámetro mayor, homogéneo y blanquecino (Fig. 1). El tejido esplénico restante era de aspecto congestivo sin mostrar patología alguna. El análisis microscópico fue revelador de una proliferación de células fusocelulares y miofibroblásticas (Fig. 2), con positividad en los estudios inmunohistoquímicos para actina y negatividad para ALK 1 y VEB, siendo el diagnóstico anatomopatológico de pseudotumor inflamatorio del bazo. El paciente evolucionó de forma favorable permaneciendo, desde entonces, asintomático.

\section{DISCUSIÓN}

Si bien la causa del pseudotumor inflamatorio del bazo es desconocida, se han investigado diversas teorías etiológicas entre las que destacamos: los agentes infecciosos y especialmente el virus de Epstein-Barr (2,3,9-11); de naturaleza vas-

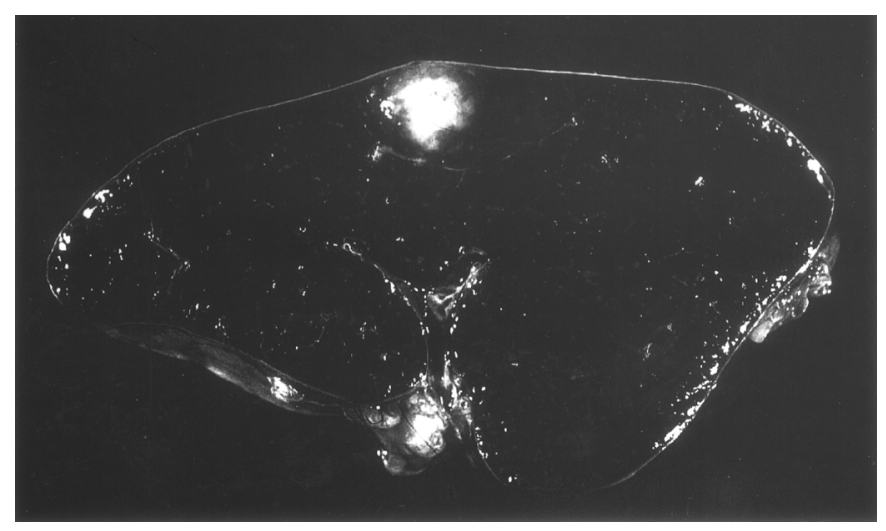

Fig. 1. Nódulo solitario bien delimitado de coloración blanquecina y consistencia firme.

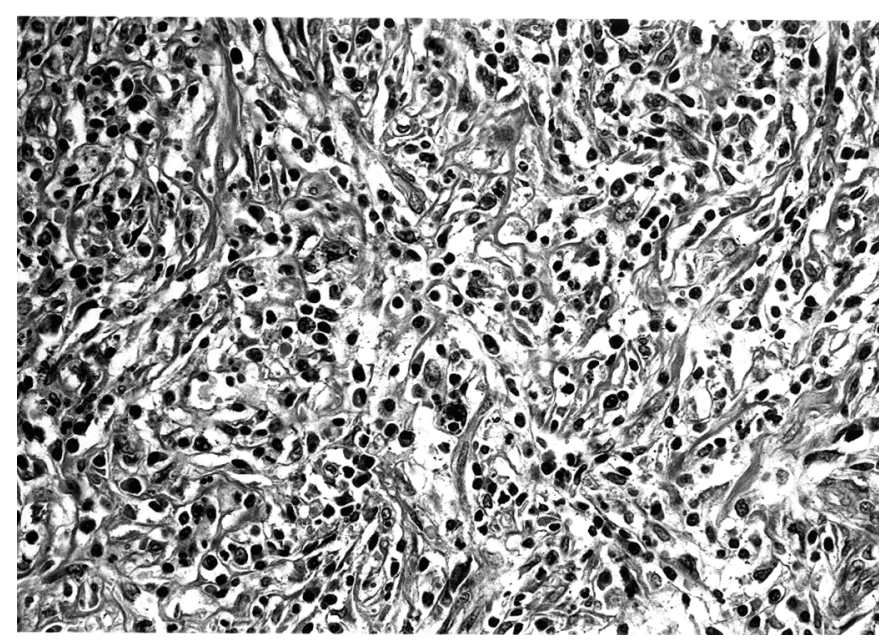

Fig. 2. Células fusiformes con núcleos ovales de apariencia citológica benigna, mezcladas con vasos sanguíneos, linfocitos y células plasmáticas.

cular, ante la presencia en la lesión de venas dilatadas y trombos vasculares; y, por último, de origen autoinmune ante la descripción de casos que cursaron con asociación a procesos autoinmunes tales como la púrpura trombocitopénica idiopática $(12,13)$. En nuestro caso, aunque los estudios para VBE en la pieza de esplenectomía fueron negativos, parece probable su implicación en la patogénesis del pseudotumor inflamatorio ya que el paciente tenía historia previa de infección por VEB.

El pseudotumor inflamatorio del bazo aparece con mayor frecuencia en torno a la $2^{\mathrm{a}}$ y $3^{\mathrm{a}}$ década de la vida, habiéndose descrito por igual en ambos sexos. Ocasionalmente, se han descrito casos de tumores en varias localizaciones a la vez como bazo e hígado (14), y bazo y ganglios linfáticos (6). Clínicamente, se manifiesta con dolor abdominal y fiebre, acompañándose de otros síntomas inespecíficos como pérdida de peso y astenia aunque, también ha habido casos de curso asintomático, siendo constante la esplenomegalia en la exploración física (Tabla I). Los estudios de laboratorio pueden aportar una alteración de las series hematológicas, principalmente anemia y trombopenia, que se relacionan con secuestro esplénico habiéndose encontrado casos de asociación con púrpura 
TABLA I

CARACTERÍSTICAS CLÍNICAS DEL PSEUDOTUMOR INFLAMATORIO DEL BAZO EN ALGUNOS ESTUDIOS DE LOS ÚLTIMOS 10 AÑOS

\begin{tabular}{|c|c|c|c|c|c|c|}
\hline & \multirow[t]{2}{*}{$n$} & \multirow{2}{*}{$\begin{array}{l}\text { Edad media } \\
\text { (rango) }\end{array}$} & \multicolumn{2}{|c|}{ Sexo } & \multirow{2}{*}{$\begin{array}{l}\text { Presentación } \\
\text { clínica }\end{array}$} & \multirow{2}{*}{$\begin{array}{l}\text { Tamaño del } \\
\text { pseudotumor, } \\
\text { cm (rango) }\end{array}$} \\
\hline & & & Varón & Mujer & & \\
\hline $\begin{array}{l}\text { Thomas et al, } \\
1993(12)\end{array}$ & 8 & $\begin{array}{c}56,6 \\
(20-87)\end{array}$ & $2(25 \%)$ & $6(75 \%)$ & $\begin{array}{l}\text { Asintomático/ } \\
\text { incidental (5), } \\
\text { dolor abdominal (2), } \\
\text { pérdida de peso (1) }\end{array}$ & $1,5-11,5$ \\
\hline $\begin{array}{c}\text { Neuhaser et al, } \\
2001 \text { (4) }\end{array}$ & 12 & $\begin{array}{c}53,0 \\
(19-77)^{*}\end{array}$ & $3 *(25 \%)$ & $8 *(75 \%)$ & $\begin{array}{c}\text { Asintomático/ } \\
\text { incidental (1), } \\
\text { dolor abdominal (5), } \\
\text { fiebre (4), } \\
\text { pérdida de peso } \\
\text { (3), otros (3) }\end{array}$ & $0,3-18$ \\
\hline $\begin{array}{l}\text { Kutok et al, } \\
2001 \text { (3) }\end{array}$ & 4 & $\begin{array}{c}50,5 \\
(37-67)\end{array}$ & $1(25 \%)$ & $3(75 \%)$ & $\begin{array}{c}\text { Asintomático/ } \\
\text { incidental (3), } \\
\text { náuseas y } \\
\text { vómitos (1) }\end{array}$ & $1,0-8,0$ \\
\hline
\end{tabular}

$\mathrm{n}=$ número de casos; ${ }^{*}$ no indicados en 1 caso

trombocitopénica autoinmune $(1,3,4,10,12)$. Nuestro caso cursó con cuadro febril acompañado de sintomatología inespecífica y esplenomegalia destacando, analíticamente, la presencia de trombopenia.

Los hallazgos radiológicos (ecografía abdominal, TAC abdominal) suelen ser expresivos y confirman la existencia de una masa localizada a nivel esplénico. Sin embargo, estas pruebas no son patognomónicas y ello obliga a elaborar tanto una adecuada valoración clínica, como un diagnóstico diferencial con otras entidades, sobre todo con procesos linfoproliferativos y neoplasias hematológicas. Otras patologías que se incluyen son el harmatoma, quistes parasitarios, epiteliales o traumáticos y enfermedades granulomatosas como la sarcoidosis $(1,7-9,12,15,16)$. En el presente caso, las pruebas radiológicas no nos hicieron sospechar su diagnóstico preoperatorio por lo que fue necesaria la intevención quirúrgica para su confirmación.

El diagnóstico del pseudotumor inflamatorio del bazo es, fundamentalmente, anatomopatológico. En casi la mitad de los casos, su diagnóstico es incidental en el estudio de otros procesos $(3,4,10,12)$. Macroscópicamente, se caracteriza por la presencia de una lesión nodular de aspecto blanquecino, bien delimitada, que puede variar en tamaño (Tabla I) y ocasionalmente ser multinodular $(4,7,12)$. Microcópicamente, resulta peculiar el aspecto con una zona de necrosis central rodeada por histiocitos, granulomas, células gigantes y otras células inflamatorias. En el área más periférica, es típica la proliferación de células fusiformes como, por ejemplo, los fibroblastos. Generalmente no se aprecian atipias celulares ni figuras mitóticas (12).
La composición celular inflamatoria es variable, constituida, esencialmente, por linfocitos y células plasmáticas, aunque también pueden observarse neutrófilos y eosinófilos y, en algunos casos, adyacente a la lesión, venas dilatadas y trombosis con fibrina. En el estudio inmunofenotípico predominan las células $\mathrm{T}$, mientras que las células $\mathrm{B}$ son poco frecuentes. Además, se llegan a contemplar histiocitos $(4,10,12)$.

En el pseudotumor inflamatorio, según la fase evolutiva de la enfermedad, el componente celular puede cambiar, por lo que se ha establecido una clasificación en tres patrones básicos: xantogranulomatoso, cuando prevalece el componente histiocitario; granuloma de células plasmáticas, si sobresalen las células plasmáticas y; pseudotumor esclerosante, ante marcados eventos escleróticos (17).

El estudio inmunohistoquímico es útil para diferenciar las diversas entidades que engloba el pseudotumor inflamatorio. Así el tumor miofibroblástico inflamatorio, formado por una proliferación de células fusocelulares y miofibroblásticas, usualmente, se comporta de forma inmunorreactiva frente a actina y vimentina de músculo liso y negativas frente a ALK 1 $(3,4)$. En nuestro caso, dada la composición celular y los resultados obtenido en los estudios inmunohistoquímicos, lo podemos considerar como tumor miofibroblástico inflamatorio.

En cuanto al tratamiento, éste es quirúrgico aconsejándose la práctica de esplenectomía, tras la cual no se han descrito casos de recurrencia ni desarrollo posterior de neoplasias hematológicas (4). No obstante, según algunos autores, en caso de obtener el diagnóstico sin haber realizado extirpación de la tumoración, se puede optar inicialmente por una actitud conservadora (14). 
A modo de conclusión, destacamos la rareza de esta entidad. Así mismo, debido a las características del pseudotumor inflamatorio del bazo, debemos subrayar la importancia de un diagnóstico diferencial con otros procesos especialmente de tipo linfoproliferativo y que, en el presente caso, aunque los estudios inmunohistoquímicos para VEB en la muestra de esplenectomía fueron negativos, dado el antecedente de mononucleosis con demostrada positividad reciente para VEB es muy probable su implicación en la patogénesis del presente pseudotumor inflamatorio del bazo.

\section{Bibliografía}

1. Monforte-Muñoz H, Ro JY, Manning JT, Landon G, Del Junco G, Carison TS, et al. Inflammatory pseudotumor of the spleen. Report of two cases with a review of the literature. Am J Clin Pathol 1991; 96: 491-5.

2. Cheuk W, Chan JKC, Shek TWH, Chang JH, Tsou MH, Yuen NWF, et al. Inflammatory pseudotumor-like follicular dendritic cell tumor. A distinctive low-grade malignant intra-abdominal neoplasm with consistent Epstein-Barr virus association. Am J Surg Pathol 2001; 25: 721-31.

3. Kutok JL, Pinkus GS, Dorfman DM, Fletcher CDM. Inflammatory pseudotumor of lymph node and spleen: an entity biologically distinct from inflammatory myofibroblastic tumor. Hum Pathol 2001; 32: 1382-7.

4. Neuhaser TS, Derringer GA, Thompson LDR, Fanburg-Smith JC, Aguilera NSI, Andriko JA, et al. Splenic inflammatory myofibroblastic tumor (inflammatory pseudotumor): a clinicopathologic and immunophenotypic a clinicopathologic and immunophenotypic study of 12 cases. Arch Pathol Lab Med 2001; 125: 379-85.

5. Nakanuma Y, Tsuneyama K, Masuda S, Tomioka T. Hepatic inflammatory pseudotumor associated with chronic cholangitis: report of three cases. Human Pathol 1994; 25: 86-91.

6. Moran CA, Suster S, Abbondanzo SL. Inflammatory pseudotumor of lymph nodes: a study of 25 cases with emphasis on morphological heterogeneity. Hum Pathol 1997; 28: 332-8.

7. Sheahan K, Wolf BC, Neiman RS. Inflammatory pseudotumor of the spleen: a clinicopathologic study of three cases. Hum Pathol 1998; 19: 1024-9.

8. Cotelingam JD, Jaffe ES. Inflammatory pseudotumor of the spleen. Am J Surg Pathol 1984; 8: 375-80.
9. Dalai BI, Greenberg H, Quiñonez GE, Gough JC. Inflammatory pseudotumor of the spleen. Morphological, radiological, immunophenotypic and ultraestructural features. Arch Pathol Lab Med 1991; 115: 1062-4.

10. Arber DA, Kamel OW, van de Rijn M, Davis RE, Medeiros LJ, Jaffe ES, Weiss LM. Frequent presence of the Epstein-Barr virus in inflammatory pseudotumor. Hum Pathol 1995; 26: 1093-8.

11. Arber DA, Weiss LM, Chang KL. Detection of Epstein-Barr virus in inflammatory pseudotumor. Semin Diagn Pathol 1998; 15: 155-160.

12. Thomas RM, Jaffe ES, Zarate-Osorno A, Medeiros LJ. Inflammatory pseudotumor of the spleen: a clinicopathologic and immunophenotypic study of eight cases. Arch Pathol Lab Med 1993; 117: 921-6.

13. Bertolín Bernades RJ, Ferrando-Marco J, Arilla Morell MJ, PallasRegueira A. Pseudotumor inflamatorio de bazo: presentación de un caso con clínica trombopénica y revisión de la literatura. An Med Interna (Madrid) 2003; 20: 330-2.

14. Di vita G, Soresi M. Patti R, Carrocio A, Leo P, Franco V, et al. Concomitant inflammatory pseudotumor of the liver and spleen. Liver 2001; 21: 217-22.

15. Wiernik PH, Rader M, Becker NH, Morris SF. Inflammatory pseudotumor of spleen. Cancer 1990; 66: 597-600

16. Franquet T, Montes M, Aizcorbe M, Barberena J, Ruia de Azua Y, Cobo F. Inflammatory pseudotumor of the spleen: ultasound and computed tomographic findings. Gastrointest Radiol 1989; 14: 181-3.

17. Someren A. Inflammatory pseudotumor of liver with occlusive phlebitis. Report a case in a child and review of the literature. Am J Clin Pathol 1978; 69: 176-81. 